$1927, \mathrm{Nr} .5)$, according to which this treatment is worthy of every consideration; similarly the criticism of Gifford, according to which the value of the method is considerable and definite.

This applies in our opinion above all-(1) to iritis glaumatosa, and (2) to glaucoma cases unsuccessfully operated on or with but transitory success.

\title{
A KNIFE FOR CORNEAL GRAFTING
}

BY

\section{H. B. Stallard}

LONDON

AN essential part of the modern technique of corneal grafting is the cutting of a shelf through the deeper layers of the cornea in order to prevent the graft from slipping into the anterior chamber of the recipient's eye. It is desirable that as far as possible the shelving of the donor's graft and the corneal bed of the recipient should correspond.

Tudor Thomas uses scissors to cut this shelf after the trephine has entered the anterior chamber. However skilful the operator these cuts with the scissors are at the best rough and irregular and the adjacent tissues are crushed and mutilated to some extent.

On this account I felt prompted to design and try a small corneal knife, a diagram of which is shown below.

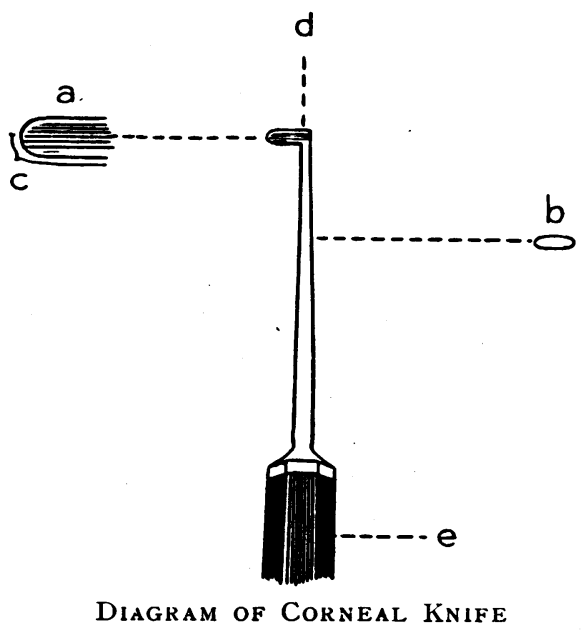

(a) Magnified view of the knife blade. The cutting edge is $1.5 \mathrm{~mm}$. long and is continued half way round the curved extremity of the knife, shown in the diagram by the bracket (c). (b) Is the shaft, which in section is a flat oval. (d) The edge (d) is rounded and smooth. (e) Bakelite handle. 
A small blade $1.5 \mathrm{~mm}$. long and $1 \mathrm{~mm}$. deep is set at right angles to the shaft which is a flat oval in section and fixed into a bakelite handle. The cutting edge is on the upper side of the knife and the upper half of the rounded extremity is sharpened. The remainder is rounded and smooth.

The knife has been tried on a few animals' eyes and in one case on the human. The technique adopted in the latter case was briefly as follows. The recipient's eye was anaesthetized with pantocain and the subconjunctival tissues injected with novocain. The conjunctiva was incised all round the limbus and undermined to an extent necessary to draw a complete conjunctival flap over the cornea and the graft. A purse-string suture was threaded $2 \mathrm{~mm}$. distal to the cut margin of the conjunctiva and at the end of the operation drawn together and tied.

In cutting the graft and preparing its bed in the recipient's eye half the corneal thickness was cut cleanly through at right angles to the surface by giving a few sharp turns to the trephine. The trephine was then tilted towards the nasal side and approximately one-third of the circumference cut through in a shelving manner into the anterior chamber. The corneal knife was then passed through the trephine incision into the anterior chamber on the flat and gently turned into a position where the shaft lay against the undivided part of the cornea and the blade was placed at an oblique angle to cut through its deeper layers from within outwards, the incision on the inner surface of the cornea being nearer its centre than the original trephine cut on the surface. By a series of firm, short, sweeping cuts in this oblique plane the deeper layers of the cornea were incised, the short incisions joining the original tre- phine incision about half way through the cornea. The knife should not be pulled through the cornea. A gentle but firm sliding action of the cutting edge divides its deeper layers quite satisfactorily until the last 1.5 to $2 \mathrm{~mm}$. or so of undivided cornea remain. In the case of the donor's cornea the graft is best separated by an oblique cut with iridotomy or de Wecker's scissors at this point as the technical difficulties of cutting with a knife so mobile an object as an almost completely separated corneal disc are considerabie. In the case of the recipient's opaque corneal disc this final severance may be effected more easily by grasping the disc opposite the point where the incision is to be made with a pair of fine, toothed, straight iris forceps thereby steadying the disc. This procedure is of course undesirable in the case of the donor's corneal graft which should not be handled with forceps.

The imperfections of this technique with the corneal knife are that the incisions through the deeper layers of the cornea are set side by side at a small angle instead of the ideal even curve but where the incisions are so small, probably $1 \mathrm{~mm}$. or less, this seems to be of little practical importance. 
It is probable that some better method than this will be devised but it appealed to me as being less likely to cause as much trauma to the deeper parts of the cornea as is the case where scissors are used to effect the necessary shelf.

In the one human case the graft fitted neatly, has taken, and the anterior chamber reformed satisfactorily without adhesion of the iris or lens capsule to the cornea.

\section{ANNOTATIONS}

\section{Classification of the Causes of Blindness}

An important report on this subject was presented at the General Assembly of the International Association for the Prevention of Blindness in April, 1935, by Professor van Duyse, of Ghent. After a brief review of the schemes in use in such countries as Germany, Belgium, France, Great Britain and the United States, the reporter gave the proposals of his organization which may be summarized as follows :-

It is proposed to consider as socially blind (a) any person who is unable to count fingers against a dark background at a distance of one metre, under good lighting conditions, after correction of the refraction, the condition of which cannot be improved. $(b)$ Persons are to be considered as blind whose visual fields are seriously impaired; (concentric narrowing down to $5^{\circ}$; disseminated scotomata; loss of field except for a strip or peripheral sector); and persons with night blindness and narrowing of the visual fields.

The classification must include the totally and the surially blind. Curable cases, and the blind in one eye are not to be included. Only the final cause of blindness is to be stated, for if primary and secondary causes leading to blindness be included, the registration forms would be too complicated.

The examination of the blind should be entrusted exclusively to an ophthalmic surgeon. Incurability must be recognized on the strength of the report of one or of several ophthalmologists.

The age of the individual is of importance, for the chances of being (not becoming) blind increase with years.

With regard to the form of classification opinions differ: some hold that a detailed classification alone is sufficiently accurate for scientific purposes; others think that an extensive classification would lead to unnecessarily complicated forms which would not commend themselves for general use. 